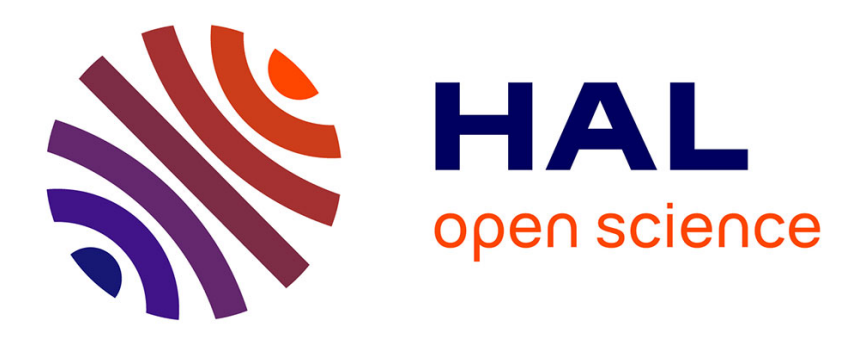

\title{
Sur la limitation de la propagation de la décharge dans les compteurs G.-M.
}

E. Picard, A. Rogozinski

\section{To cite this version:}

E. Picard, A. Rogozinski. Sur la limitation de la propagation de la décharge dans les compteurs G.-M..

J. Phys. Radium, 1953, 14 (5), pp.304-306. 10.1051/jphysrad:01953001405030400 . jpa-00234737

\section{HAL Id: jpa-00234737 https://hal.science/jpa-00234737}

Submitted on 1 Jan 1953

HAL is a multi-disciplinary open access archive for the deposit and dissemination of scientific research documents, whether they are published or not. The documents may come from teaching and research institutions in France or abroad, or from public or private research centers.
L'archive ouverte pluridisciplinaire HAL, est destinée au dépôt et à la diffusion de documents scientifiques de niveau recherche, publiés ou non, émanant des établissements d'enseignement et de recherche français ou étrangers, des laboratoires publics ou privés. 


\title{
SUR LA LIMITATION DE LA PROPAGATION DE LA DÉGHARGE DANS LES GOMPTEURS G.-M.
}

\author{
Par E. PICARD et A. ROGOZINSKI, \\ Division des Constructions électriques, \\ Commissariat à l'Énergie atomique, Saclay.
}

\begin{abstract}
Sommaire. - On décrit un montage à réaction, comportant une seule double triode, qui permet de confiner la décharge se produisant dans un compteur à une portion limitée du fil. La réaction survient dans un temps inférieur à $1^{-7} \mathrm{~S}$ après le début de la décharge et se prolonge pendant environ ${ }^{2} \cdot 1^{-6} \mathrm{O}^{-6}$. Les avantages qui découlent de la limitation de la décharge sont : une augmentation de la vie du compteur, une diminution de son temps mort et une réduction du nombre d'impulsions parasites.
\end{abstract}

1. Dans les conditions habituelles d'emploi des compteurs G.-M. à autoextinction, on n'utilise généralement qu'une fraction de l'impulsion obtenue et l'on se soucie peu du sort de la décharge qui continue à se développer le long du fil du compteur. Le gaspillage d'un nombre élevé de molécules du gaz extincteur qui en résulte provoque inutilement les effets, indésirables suivants :

a. une diminution de la vie du compteur;

$b$. une augmentation du temps mort et un accroissement des pertes dans le cas de taux de comptage élevés;

c. un accroissement de la probabilité d'émission secondaire de la cathode, proportionnelle au nombre d'ions positifs qui y parviennent : d'où production d'impulsions parasites, qui sont à l'origine de la pente du palier du compteur, et réduction de longueur de ce palier.

Ces effets sont d'autant plus accusés que la longueur du compteur est plus grande.

Plusieurs auteurs, Simpson [1], Hodson [2], Collinge [3] et Cosyns [4] ont cherché à atténuer ces effets, en particulier, en renversant le signe de la tension appliquée au compteur pendant un bref intervalle de temps après l'apparition de l'impulsion. En procédant de la sorte, ils ont cherché non pas tant à limiter la perte de molécules, mais plutôt à détourner les ions positifs de la cathode. Les circuits établis dans ce but sont fort compliqués.

2. Nous avons abordé ce prob̀lème sous un angle plus direct et cherché sa solution en provoquant un arrêt de la décharge, dès que l'amplitude de l'impulsion a atteint une valeur suffisante pour actionner les circuits contrôlés par le compteur. Une solution du même genre a déjà été adoptée par Elliot [5] et par Den Hartog et Muller [6] et, tout récemment, des résultats intéressants ont été obtenus par Porter et Ramsey [7] sur un sujet analogue.

Pour parvenir au résultat cherché, nous avons utilisé un monovibrateur rapide (fig. I) dont le mode d'action est le suivant : dès que l'impulsion du compteur dépasse le seuil de sensibilité du monovibrateur, celui-ci est déclenché et réagit sur le fil du compteur en éteignant la décharge; on obtient ainsi (fig. 2) la courbe rectangulaire II, de largeur $\theta$ aisément ajustable, au lieu de la courbe I, observée sur le fil du

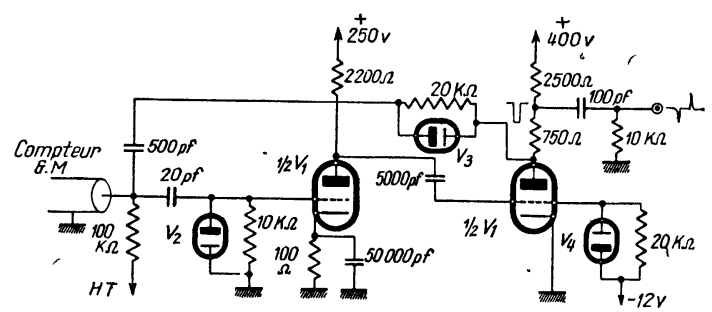

Fig. I. - Détails du circuit de limitation de la décharge.

$\mathrm{V}_{1}=$ double triode ECC $81 ; \mathrm{V}_{2}, \mathrm{~V}_{3}$ et $\mathrm{V}_{4}=$ diodes au germanium $\mathrm{OA}$ 53. Les diodes $\mathrm{V}_{2}$ et $\mathrm{V}_{4}$ servent à accélérer le retour du circuit à son état initial dans le cas des taux de comptage élevés. La résistance de $20 \mathrm{k} \Omega$, qui est branchée aux bornes de la diode $\mathrm{V}_{3}$, sert à augmenter la sensibilité du circuit, en empêchant une partie de l'impulsion fournie par le compteur de s'écouler à travers la capacité de $500 \mathrm{pF}$. Mais dès que la réaction s'amorce, cette résistance se trouve-pratiquement court-circuitée par $\mathrm{V}_{3}$.

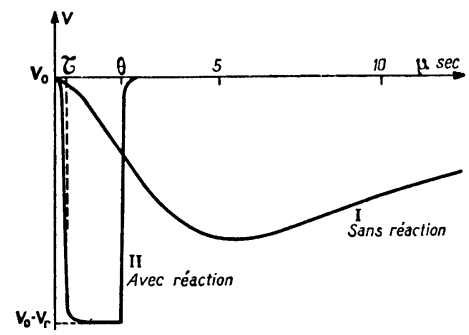

Fig. 2. - Allure des impulsions sans et avec réaction.

Le circuit à réaction commence à agir iaprès un temps $\tau$ inférieur à ${ }_{1} 0^{-7} \mathrm{~s}$, lorsque l'impulsion a atteint quelques dixièmes de volts. Celle-ci prend alors la forme imposée par le monovibrateur rapide (courbe II). En l'absence du circuit à réaction, l'impulsion obtenue est celle de la courbe I.

compteur en l'absence de toute réaction. Le commencement de la réaction survient dans un temps $\tau$ très bref, inférieur à $\mathrm{IO}^{-7} \mathrm{~s}$, après le déclenchement de la décharge dans le compteur et permet de bien définir 
le début de l'impulsion. D'autre part, le front de l'impulsion de réaction est suffisamment raide et son amplitude $V_{r}$ suffisamment grande - elle peut atteindre plusieurs centaines de volts - pour que la chute de tension provoquée sur le fil arrête immédiatement le développement de la décharge dont la propagation se limite alors à une longueur $l=v \tau \sim 5 \mathrm{~mm}$, puisque la vitesse de propagation $v$ de la décharge est de l'ordre de $5.10^{-6} \mathrm{~cm} . \mathrm{s}^{-1}$.

Bien entendu, pour une certaine valeur $V_{r}$ de la tension de réaction, on utilisera toujours une surtension $V_{s}$ au-dessus du seuil du compteur un peu inférieure à $V_{r}$. En effet, dès que l'impulsion du compteur dépasse une valeur de l'ordre de quelques dizièmes de volt, la réaction s'amorce et ramène la tension aux bornes du compteur de la tension de repos $V_{0}$ à $V_{0}-V_{r}$ dans un laps de temps de l'ordre de $2 . \mathrm{Io}^{-7} \mathrm{~s}$, déterminé par la pente $\mathrm{du}$,front de l'impulsion de réaction. $\mathrm{Si} V_{r}>V_{s}$, l'avalanche s'arrêtera. Dans le cas contraire, la décharge continuera à se développer et à se propager. En effet, pendant la durée de l'impulsion de réaction, la multiplication des charges subsistera, bien qu'à un taux inférieur au taux normal, mais elle reprendra sa valeur initiale, aussitôt que cette impulsion aura passé. On obtient ainsi des caractéristiques illustrées par la figure 3 , dans laquelle la courbe en trait plein correspond au circuit normal sans réaction et la courbe en pointillés au circuit étudié pour $V_{r}=3$ oo Volts.

On constate effectivement que l'application de la réaction entraîne une augmentation de la longueur du palier et une augmentation de comptage d'autant plus importante que le taux de comptage est lui-

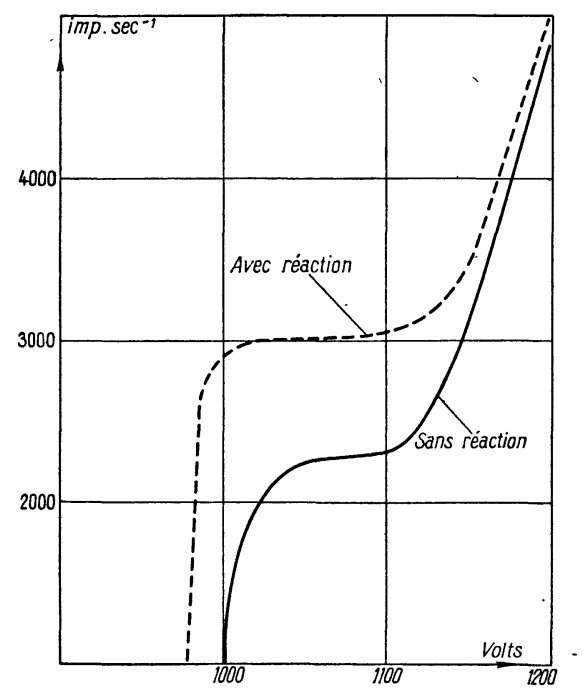

Fig. 3. - Paliers d'un compteur usagé, obténus avec et sans circuit de limitation de la décharge.

On notera l'allongement $\mathrm{du} \cdot$ palier et l'augmentation du comptage résultant de la diminution du temps mort, lorsqu'on passe du premier au second montage (comparer avec la figure 4). même plus élevé. Cette augmentation résulte de la diminution du temps mort du compteur.

Notons que dans la plupart des cas on n'aura aucun intérêt à travailler avec des valeurs de $V_{s}$ très élevées et que l'on pourra ainsi se borner à des tensions $V_{r} \simeq$ Ioo Volts.

3. Les mesures effectuées avec le dispositif décrit nous ont permis de vérifier que les trois effets énumérés plus haut se trouvaient nettement

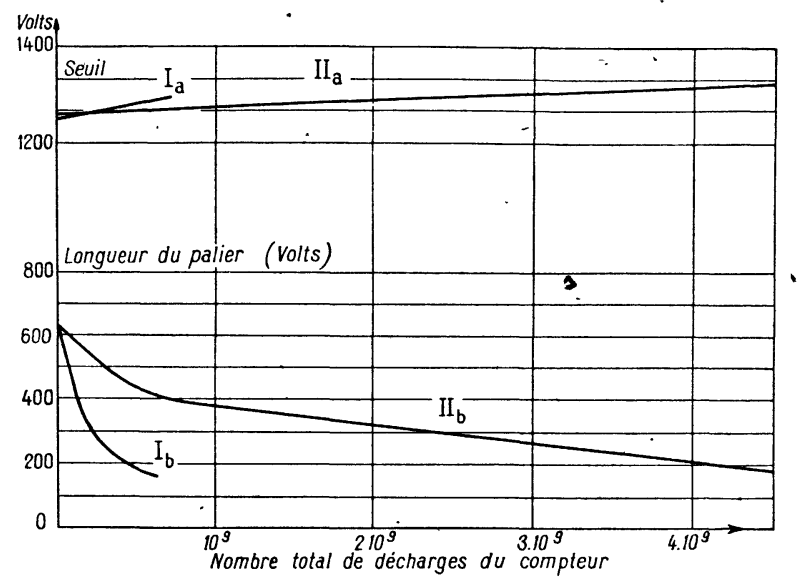

Fig. 4. - Évolution du seuil (courbes $\mathrm{I}_{a}$ et $\mathrm{II}_{u}$ ) et du palier (courbes $\mathrm{I}_{b}$ et $\mathrm{II}_{b}$ ), en fonction du nombre total de décharges du compteur.

La longueur du palier est définie par la différence des deux surtensions pour lesquelles le taux de comptage s'écarte de ro pour i oo de part et d'autre du milieu du palier. Les courbes $I_{a}$ et $I_{b}$ correspondent 'à un compteur sans circuit de limitation de la décharge, et. les courbes II $_{a}$ et $\mathrm{II}_{b}$ à un compteur du même type mais dont la décharge a été limitée.

atténués. C'est ainsi que, en soumettant à un rayonnement intense (2.1 $0^{4}$ imp. $\left.\mathrm{s}^{-1}\right)$ deux compteurs, ayant des caractéristiques aussi voisines que possible, associés respectivement à un circuit sans et avec réaction, on constate une évolution plus rapide du seuil et de la longueur du pälier du premier compteur (fig. 4).

On obtient, d'autre part, une réduction très appréciable du temps mort du compteur dont la mesure était effectuée par une méthode des coïncidences retardées, qui sera décrite ultérieurement [8].

On trouve qu'avec le type de compteur utilisé (diamètre $25 \mathrm{~mm}$, longueur utile $5 \mathrm{o} \mathrm{cm}$; remplissage en $\mathrm{cm} \mathrm{Hg}: \mathrm{He}, 60 ; \mathrm{A}, \mathrm{o}, 4$; alcool, $\mathrm{I}, 2)$ le temps mort $n$ passe de $190 \mu \mathrm{s}$ à une valeur $\eta_{r}$ inférieure à ıo $\mu \mathrm{s}$. Cette réduction $\Delta n=n-\eta_{r}$ est due à ee qu'une faible tranche du compteur, déterminée approximavement par $l$, se trouve affectée par la décharge et présente un temps mort habituel, tandis que le reste du compteur demeure dans des conditions normales de fonctionnement. Dans ce cas particulier, les pertes respectives sont de io et 0,2 pour ioo pour un taux de comptage de $10^{3} \mathrm{imp} . \mathrm{s}^{-1}$. 
Une méthode graphique très simple permet de on obtient trouver rapidement la valeur de $\Delta n$. On a

$$
n_{0}=\frac{n}{\mathrm{I}-\eta n}=\frac{n_{r}}{\mathrm{I}-\eta_{r} n_{r}}
$$

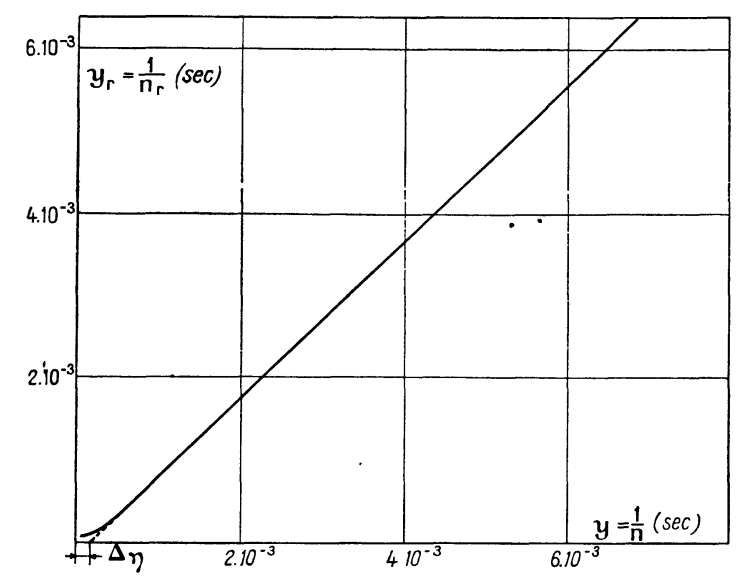

Fig. 5. - Détermination graphique de $\Delta \eta=\eta-\eta_{r}$.

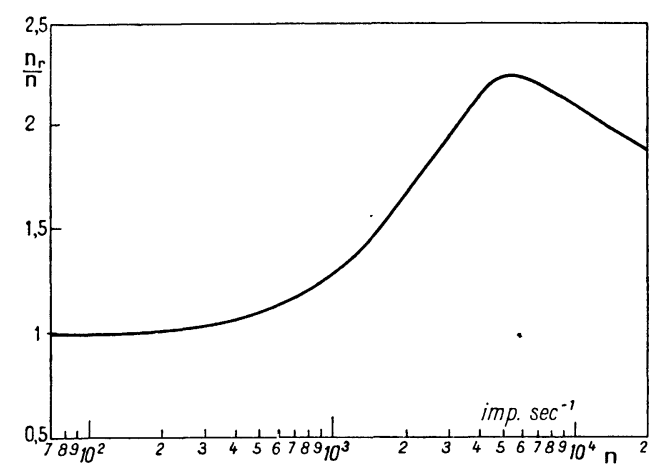

Fig. 6. - Courbe de $\frac{n_{r}}{n}$ en fonction de $\eta_{\text {. }}$

Elle illustre l'influence du dircuit décrit sur la récupération d'une proportion notable d'impulsions perdues avec un circuit normal. La partie décroissante de la courbe est due au fait bien connu de la diminution du temps mort d'un compteur aux comptages élevés.

où $n$ et $n_{r}$ représentent, respectivement, les taux de comptage réels, mesurés sans et avec réaction, et $n_{0}$ le taux que l'on aurait obtenu, si $n$ était nul. En posant

$$
y=\frac{\mathrm{I}}{n} \quad \text { et } \quad y_{r}=\frac{\mathrm{I}}{n_{r}},
$$

$$
y_{r}=y-\left(\eta-\eta_{r}\right) .
$$

On trace $y_{r}$ en fonction de $y$ et l'on extrapole la droite ainsi obtenue jusqu'à son intersection avec l'axe des $y$. L'abscisse à l'origine donne alors directement $\Delta \eta$ (fig. 5).

On remarquera que pour des valeurs de $y$ supérieures à $0,67 \cdot 10^{-3} \mathrm{~s}$, c'est-à-dire pour des taux de comptages $n$ inférieurs à $1,5.1^{3} \mathrm{imps}^{-1}$, les points se placent sur une ligne droite, ce qui signifie que $\Delta n$ conserve une valeur constante dans cette région. Pour des comptages supérieurs, $\Delta n$ subit une diminution marquée. Comme on a toutes raisons de croire que $n_{r}$ demeure constant, on peut en déduire que c'est $n$ qui diminue avec des taux de comptages croissants. Ce fait est d'ailleurs en accord avec les résultats signalés par d'autres auteurs (Collinge [3]), ainsi qu'avec nos propres résultats obtenus à l'aide d'un dispositif à coïncidences retardées [8]. La courbe de la figure 6 représente $\frac{n_{r}}{n}$ en fonction de $n$. Elle illustre l'influence du circuit décrit sur la récupération d'une proportion notable des impulsions perdues avec un circuit normal. Cette récupération provient évidemment de la réduction $\Delta \eta$ du temps mort. Pour $n<\simeq 2 . \mathrm{Io}^{2} \mathrm{imp} . \mathrm{s}^{-1}, n \simeq n_{r}$ : l'effet de récupération est négligeable, car les pertes le sont également. Cet effet s'accentue jusqu'à $n \simeq 5.10^{3} \mathrm{imp} . \mathrm{s}^{-1}$; mais pour des comptages encore plus élevés, on observe une réduction, déjà mentionnée, de $n$, ce qui entraîne en même temps une atténuation sensible de l'effet de récupération.

Signalons, enfin, un autre avantage de la forme d'impulsion obtenue : en différentiant le front arrière de celle-ci, on obtient immédiatement une impulsion d'amplitude appréciable, retardée de 0 . Le montage décrit permet ainsi de simplifier sensiblement des circuits comportant des coïncidences retardées.

4. On peut affirmer, en conclusion, que le montage décrit, limitant la décharge du compteur et qui ne comporte qu'une seule double triode, conduit à une amélioration importante des caractéristiques des compteurs G.-M.

BIBLIOGRAPHIE.

[1] Simpson J. A. - Phys. Rev., i $944,66,39$.

[2] Hodson A. L. - J. Sc. Inst., i 948,25 , 1 .

[3] Collinge B. - Proc. Phys. Soc., G. B., I95o, 63, I 5.

[4] Cosyns M. - Communication privée.

[5] Elliot H. - Proc. Phys. Soc., G. B., i 949, 62, 369.
[6] Den Hartog H. et Muller F. A. - Physica, r 950, 16, 26.

[7] Porter W. C. et Ramsey W. E. - J. Frank. Inst., 1952,254, i 53.

[8] Picard E. et Rogosinski A. - J. Physique Rad. (sous presse). 